adverse events $(n=3)$. Four out of 9 RCTs demonstrated a significant increase in stool frequency following fibre consumption compared to placebo. Stool consistency significantly improved following fibre consumption in 2 RCTs, while 4 RCTs failed to show an effect. Three RCTs demonstrated a significant increase in stool weight, but 2 RCTs did not. Bifidobacteria significantly increased in 2 out of 3 RCTs (inulin and one GOS intervention). No side effects were observed and no patient withdrew because of adverse events. Attrition bias was high amongst trials, while selection bias and performance bias were unclear due to inadequate reporting. Outcome data will undergo meta-analysis.

Conclusion Current guidelines recommend the use of fibre as first-line treatment for constipation. However, this review shows that not all studies support its use in adults. Most studies suffer from small sample sizes and poor design with high risk of bias. The paucity of high quality data highlights the need for further large, methodologically rigorous RCTs.

Disclosure of Interest S. Christodoulides Grant/research support from: Nestec Ltd, E. Dimidi Grant/research support from: Nestec Ltd, K. Fragkos: None Declared, P. McLean Employee of: Nestec Ltd, Q. Aziz: None Declared, K. Whelan: None Declared, S. M. Scott: None Declared.

\section{PWE-185 BELIEFS ABOUT MANAGEMENT OF IRRITABLE BOWEL SYNDROME IN PRIMARY CARE: CROSS-SECTIONAL SURVEY}

UN Shivaji*, AC Ford. Leeds Gastroenterology Institute, St. James's University Hospital, Leeds, UK

\subsection{6/gutjnl-2014-307263.445}

Introduction There have been considerable advances in evidence synthesis concerning management of irritable bowel syndrome (IBS) in the last 5 years, with guidelines for its management in primary care published by the National Institute of Health and Care Excellence (NICE). We examined beliefs about IBS management among primary care physicians.

Methods This was a cross-sectional web-based questionnaire survey of 275 primary care physicians registered with three clinical commissioning groups in Leeds, UK. We e-mailed a link to a SurveyMonkey questionnaire, containing 18 items, to all eligible primary care physicians. Participants were given 1 month to respond, with a reminder sent out after 2 weeks.

Results One-hundred and two (37.1\%) primary care physicians responded. Eighty-four (82.4\%) of the respondents confirmed that they used clinical symptoms or signs elicited during the history and physical examination to diagnose IBS, with only 10 (9.8\%) using the Rome criteria, and 4 (3.9\%) the Manning criteria. A further 4 participants stated that they referred to a Gastroenterologist to confirm the diagnosis. Seventy (68.6\%) primary care physicians agreed or strongly agreed that IBS was a diagnosis of exclusion, with only 5 (4.9\%) strongly disagreeing with this statement. More than $80 \%$ checked coeliac serology often or always in suspected IBS. Between 56\% and 76\% believed soluble fibre, antispasmodics, peppermint oil, and psychological therapies were potentially efficacious therapies (table). The respondents were less convinced that antidepressants or probiotics were effective. Despite perceived efficacy of psychological therapies, $80 \%$ stated these were not easily available. Levels of use of soluble fibre, antispasmodics, and peppermint oil were in the range of $40 \%$ to $>50 \%$. Most primary care physicians obtained up-to-date evidence about IBS management from NICE guidelines.

Conclusion Most primary care physicians still believe IBS is a diagnosis of exclusion, and many are reluctant to use antidepressants or probiotics to treat IBS. More research studies addressing diagnosis and treatment of IBS based in primary are required.

Disclosure of Interest None Declared.

\section{PWE-186 UNDER-UTILISATION OF FAECAL CALPROTECTIN TO EXCLUDE IBD IN PATIENTS WITH FUNCTIONAL BOWEL DISORDERS}

VI Astle*, NR Lewis. Nottingham Digestive Diseases Centre, Nottingham University Hospital, Nottingham, UK

\subsection{6/gutjnl-2014-307263.446}

Introduction Calprotectin is a protein released by neutrophils in response to the presence of inflammation in the bowel ${ }^{1}$. Based on secondary care data with a cut-off of $50 \mathrm{mcg}$ using ELISA assay it has a sensitivity of $93 \%$ and specificity of $94 \%{ }^{2}$ to exclude inflammatory bowel disease (IBD) from functional bowel disorders. Calprotectin can be measured in a stool sample with this non-invasive test significantly cheaper than endoscopy and associated new patient referral ${ }^{3,4}$. Faecal calprotectin (FC) has also been shown to correlate with mucosal disease activity and can help to predict response to treatment or relapse in IBD ${ }^{1}$. We report the routine use of FC in gastroenterology practice at our hospital.

Methods All FC tests performed between 01/07/12 and 31/12/ 12 were systematically collected and reason for testing determined. Endoscopic, histological, radiological, laboratory and clinical records were systematically searched to identify tests performed in patients with FC results.

Results 294 FC tests were performed during the study period: $203(69.0 \%)$ for assessment of IBD and 91 (31.0\%) tests to exclude IBD in patients with suspected functional bowel disorders. Mean age of patients with suspected functional bowel disorders was 45 (SD 16.8) years and 62.6\% were female. Of the patients with suspected IBS who had a normal FC value $(\mathrm{n}=75), 50.7 \%$

\begin{tabular}{|c|c|c|c|c|c|}
\hline & Strongly agree & Agree & Neutral & Disagree & Strongly disagree \\
\hline Soluble fibre is an effective therapy (\%) & $3(2.9)$ & $54(52.9)$ & $39(38.2)$ & $6(5.9)$ & $0(0)$ \\
\hline Antispasmodics are an effective therapy (\%) & $6(5.9)$ & $71(69.6)$ & $25(24.5)$ & $0(0)$ & $0(0)$ \\
\hline Peppermint oil is an effective therapy (\%) & $4(3.9)$ & $64(62.7)$ & $30(29.4)$ & $3(2.9)$ & $1(1.0)$ \\
\hline Probiotics are an effective therapy (\%) & $1(1.0)$ & $20(19.6)$ & $56(54.9)$ & $22(21.6)$ & $2(2.0)$ \\
\hline TCADs are an effective therapy (\%) & $2(2.0)$ & $49(48.0)$ & $42(41.2)$ & $6(5.9)$ & $1(1.0)$ \\
\hline SSRIs are an effective therapy (\%) & $0(0)$ & $33(32.4)$ & $49(48.0)$ & $17(16.7)$ & $0(0)$ \\
\hline Psychological therapies are an effective therapy (\%) & $5(4.9)$ & $55(53.9)$ & 40 (39.2) & $1(1.0)$ & $0(0)$ \\
\hline
\end{tabular}


had a colonoscopy, 20.0\% had CT or MRI and 9.3\% had a flexible sigmoidoscopy. Of the 75 patients with suspected functional bowel disorder with normal FC values, 2 patients were diagnosed with Crohn's disease affecting the terminal ileum on colonoscopy. For patients with suspected IBS the test had a specificity of $80.2 \%$ and a negative predictive value of $97.3 \%$.

Conclusion Despite normal FC result, a significant proportion of young adults with suspected functional bowel disorder undergo colonoscopy with normal test results. As other studies have shown, consideration of the FC result before further investigations are ordered can reduce the number of patients requiring endoscopy or imaging and thus reduce cost ${ }^{1}$. However, FC is less sensitive for small bowel Crohn's disease and therefore careful history taking is required to ensure this is not missed due to a negative FC result.

\section{REFERENCES}

1 Smith LA et al. World J Gastroenterol 2012 Dec 14; 18(46):6782-6789

2 Nice Guideline DG11 Faecal calprotectin diagnostic tests for inflammatory diseases of the bowel

3 NHS England National Tariff Payment System. http://www.monitor.gov.uk/NT. Accessed on 22/1/14

4 Faecal Calprotectin - Is it Requested Appropriately and is it Cost Effective? Gut2013;62:A266-A267

Disclosure of Interest None Declared.

\section{Endoscopy III}

\section{PTH-001 USE OF PANCREATIC STENTS IN ENDOSCOPIC RETROGRADE CHOLANGIO-PANCREATOGRAPHY}

${ }^{1} \mathrm{~A}$ Malik*, 'G Thomas, ${ }^{2}$ A Roberts. 'Gastroenterology, NHS, Cardiff, UK; ${ }^{2}$ Radiology, NHS, Cardiff, UK

\subsection{6/gutjpl-2014-307263.447}

Introduction The indications for placing pancreatic stents (PS) during endoscopic retrograde cholangio-pancreatography (ERCP) include pancreatic duct stones and strictures, prevention of post-ERCP pancreatitis and as an aid to biliary cannulation, where there is persistent preferential pancreatic cannulation. The flange at the proximal end of the stent used in our practice (Cook UK) is designed to prevent spontaneous migration. As a minimum, abdominal $\mathrm{x}$-ray (AXR) should be performed at 4 weeks to confirm spontaneous stent passage. The aims of this study were to assess a) indications for PS b) compliance with AXR recommendation c) whether flange removal aids spontaneous stent passage.

Methods Retrospective review of ERCP reports and case notes in 1250 ERCPs performed at our institution between August 2011 and December 2013.

Results In 82 of 1250 ERCPs, PS were placed (6.5\%). 31 male, 51 female, mean age 63 years (range 20-92).

The indication for PS placement in all 82 was to aid biliary cannulation, and in all 82, the PS was left in situ to reduce the pancreatitis risk. In $72 / 82$, biliary cannulation was successful (pre-cut in 55).

42/82 had AXR, and in 36/42 the stent had passed.

21/82 had repeat ERCP and in 15/21 the stent had passed.

19/82 (23\%) had no record of AXR or repeat ERCP.

Based on operator preference, the flange was removed in 38 and left in situ in 44. Of the 12 PS that failed to pass spontaneously the flange was removed in 4 and left in situ in 8 .

Conclusion Pancreatic stents are a useful aid to biliary cannulation, and pass spontaneously in the majority of patients, although flange removal may facilitate this. Compliance with the minimum recommendation of post-ERCP AXR to confirm stent passage in only $77 \%$ is poor, and requires further investigation.

Disclosure of Interest None Declared.

\section{PTH-002 ERCP: BALANCING DEMAND, TRAINING AND MANPOWER IN SOUTH EAST REGION}

${ }^{1}$ A Jeevagan*, ${ }^{2} \mathrm{P}$ Rai, ${ }^{3} \mathrm{G}$ Bird. ' Gastroenterology, Royal Sussex County Hospital, Brighton, UK; ${ }^{2} F Y 2$, KSS Deanery, Kent, UK; ${ }^{3}$ Gastroenterology, Maidstone Hospital, Maidstone, UK

\subsection{6/gutjnl-2014-307263.448}

Introduction The purpose of this survey is to assess trainees attitudes and exposure to ERCP training in the south east region of England and also to explore the demand for ERCP endoscopists in future.

Methods Two separate short survey questionnaires were sent using an online survey tool between July 2012 to October 2012. One was sent to the trainees and the second questionnaire was sent to the Consultant Gastroenterologists in this region. In some instances survey was conducted through telephone, e-mail or in person.

Results Out of 43 trainees, nearly 10/43(23\%) were getting trained in ERCP, 14/43 (33\%) mentioned that their training needs had been assessed, 32/43 (75\%) were not aware of the current need for consultant ERCPs in this region. Among the trainees who do not want to get trained in ERCP, 5/33 (16\%) of them had interest in other sub-specialty, 4/33(14\%) considered it a difficult procedure to get trained and 3/33(9\%) did not have any appropriate guidance. The other reasons include high risks and complication $3 / 33(9 \%)$, longer duration of training $1 / 33$ $(3 \%)$ and lack of training facilities $2 / 33(6 \%)$. Among the trainees who performed ERCPs only 2/10(20\%) had done more than 100 procedures. None of them got more than $75 \%$ selective duct cannulation rate. Majority of trainees, about 30/43 (70\%) of them pointed out that ERCP should not be an essential skill for gaining a consultant job.

Out of 55 consultants gastroenterologists 27/55 (50\%) of them perform ERCP. Of these 27 consultants, 18/27 (67\%) trained at least one trainee. Only 3/27 (11\%) consultants mentioned that they would stop doing ERCP procedures in the next 5 years and 6/27(22\%) within next 10 years. $7 / 27(25 \%)$ of the consultants thought that their colleague/colleagues would take over the ERCP service when they retire and 12/27 (45\%) thought that new ERCP consultants will be recruited.

Conclusion This survey suggests that there is currently only a need for 2-3 trainees to be learning ERCP if the current demand is stable. The number of trainees training in ERCP need to be very small unless we accept that the trained ERCP endoscopists might give up on transition to consultant. There is a disparity in that consultants claim they are delivering ERCP training but this is not reflected in the results of the trainee's responses. Most of the trainees are in their early stage of in the ERCP training. None of the trainees got over $75 \%$ selective cannulation rate. Disclosure of Interest None Declared.

\section{PTH-003 FULLY COVERED SELF EXPANDING METALS STENTS ARE EFFECTIVE IN REMODELLING BENIGN BILIARY STRICTURES IN PATIENTS WITH CHRONIC PANCREATITIS}

B Paranandi* ${ }^{*}$ D Joshi, GH El-Sayed, P Patel, MH Chapman, SP Pereira, GJ Webster, GJ Johnson. Pancreaticobiliary Medicine, University College London Hospitals, London, UK

10.1136/gutjnl-2014-307263.449 